\title{
Speckle Denoising With NL Filter and Stochastic Distances Under the Haar Wavelet Domain
}

\author{
Pedro A. A. Penna \\ Computing Department \\ Federal University of São Carlos (UFSCar) \\ Email: pedroalagao@gmail.com
}

\author{
Nelson D. A. Mascarenhas \\ Computing Department \\ Federal University of São Carlos (UFSCar) \\ Centro Universitário Campo Limpo Paulista (UNIFACCAMP) \\ Email: nelson@dc.ufscar.br
}

\begin{abstract}
Synthetic aperture radar SAR imaging systems have a coherent processing that causes the appearance of the multiplicative speckle noise. This noise gives a granular appearance to the terrestrial surface scene impairing its interpretation. The similarity between patches approach is applied by the current state-of-the-art filters in remote sensing area. The goal of this manuscript is to present a method to transform the non-local means (NLM) algorithm capable to mitigate the noise. Singlelook speckle and the NLM under the Haar wavelet domain are considered in our research with intensity SAR images. To achieve our goal, we used the Exponential-Polynomial (EP) and Gamma distributions to describe the Haar coefficients. Also, stochastic distances based on these two mentioned distributions were formulated and embedded in the original NLM technique. Finally, we present analyses and comparisons of real scenarios to demonstrate the competitive performance of the proposed method with some recent filters of the literature. ${ }^{1}$
\end{abstract}

\section{INTRODUCTION}

The image processing literature is always searching for new algorithms to attenuate noise [1] because the filters are used in a vast amount of disciplines like Forensic Science, Astronomy, Medicine and Biology [2].

Synthetic aperture radar (SAR) systems are important for monitoring the terrestrial surface activities like oil leakage in the oceans, melting glaciers and borderlands analysis. These systems are applicable in all sunlight and weather conditions [3]. Nevertheless, SAR images are contaminated with multiplicative speckle noise that emerges from the interference on the return signal given by the surface elements of the target [4]. The speckle gives a granular appearance in SAR images making their analyses and interpretations a difficult task. Furthermore this noise is present not only in SAR images, but also in medical ultrasound, sonar and laser images [5].

Based on the non-local means (NLM) filter [6], the collaborative filtering techniques like Block Matching 3D (BM3D) [7] and sparsity based methods as K-SVD [8] were developed. These techniques use the assumption of similarity between patches [9], i.e., $n x n$ windows with $n \in \mathbb{N}^{*}$ and odd, centered in the pixels of an image [4]. The similarity is given by a metric that relates the gray values of the pixels of each patch.

The filters above mentioned were originally proposed for additive white Gaussian noise (AWGN), while the Probabilistic Patch-Based filter (PPB) [10] was made for additive and

\footnotetext{
${ }^{1}$ This article is related to a Ph.D. thesis.
}

multiplicative noise. The NLM method may be applied to reduce speckle noise in (Pol)(In)SAR images [11]. One can say that SAR-BM3D [12] and FANS [13] are considered the state-of-the-art with very good results [4].

The stochastic distances [14] have already been used to remove noise in many areas: speckle in SAR images [4], [15][18] and ultrasound images [19], [20], [21] and Poisson noise in [22] and [23].

The wavelets transforms [24] have been used by many applications in areas like Astrophysics and Geophysics [25]. We present the results for the Haar wavelet, which is considered the simplest case [26]. The objective is to extend the NLM filter capability in the wavelet domain to reduce the speckle noise in intensity SAR images using the stochastic distances. This paper presents new stochastic distances based on the Gamma and Exponential-Polynomial (EP) distributions [27].

Results with real experiments show that the proposed method and distances generate competitive results with the state-of-the-art filters and other NLM based algorithms. Finally, the rest of the paper is organized as follows: Section II presents the model of speckle and the wavelets fundamentals, Section III explains the NLM filter and the stochastic distances for EP and Gamma distributions, real experiments are discussed in Section V and the proposed research is concluded with future works in Section V.

\section{SAR INTENSITY IMAGE AND THE WAVELET APPROACH}

A SAR system sends electromagnetic pulses and captures the backscattered signal of a target. Considering an intensity SAR image, the return $Z$ is the product of two independent random variables given by

$$
Z=X . Y
$$

where $X$ and $Y$ represent, respectively, the backscatter return and the speckle noise [5]. The intensity format of a SAR follows a Gamma distribution $f_{Y}(y) \sim \Gamma(L, L / \alpha)$ [28], [29] with a probability density function (pdf), expressed by [27]

$$
f_{Y}(y ; L, L / \alpha)=\frac{L^{L}}{\alpha \Gamma(L)}\left(\frac{y}{\alpha}\right)^{L-1} \exp \left(-\frac{L}{\alpha} y\right),
$$

where $L>0$ and $\alpha$ denote, respectively, the number of looks and the underlying reflectivity mean. 


\section{A. Wavelet Paradigm}

A wavelet can be expressed by [27]

$$
\psi_{a, b}(t)=\frac{1}{\sqrt{a}} \psi\left(\frac{t-b}{a}\right)
$$

where $a$ corresponds to the scale size, $b$ is the translation and $t$ denotes time.

The signal $x$ can be decomposed by [30]

$$
x(t)=\sum_{k=-\infty}^{\infty} c_{J, k} \phi_{J, t}(k)+\sum_{k-\infty}^{\infty} \sum_{j=1}^{J} \psi_{j, t}(k) w_{j, k},
$$

where

$$
\phi_{j, t}(x)=2^{-j} \phi\left(2^{-j} x-t\right)
$$

and

$$
\psi_{j, t}(x)=2^{-j} \psi\left(2^{-j} x-t\right)
$$

The $\phi$ function denotes the scale function and $\psi$ represents the wavelet. The $j$ parameter indicates the number of resolutions for decomposition. The coefficients are expressed by:

$$
\begin{aligned}
& c_{j+1, t}=\sum_{k-\infty}^{\infty} h(k-2 t) c_{j, k}, \\
& w_{j+1, t}=\sum_{k-\infty}^{\infty} g(k-2 t) c_{j, k} .
\end{aligned}
$$

$w_{j, t}$ is the wavelet coefficient and $c_{j, t}$ coefficient represents a smoothed version of the original signal from the scale function. The $h$ and $g$ terms represent, respectively, the lowpass and high-pass impulse response of the filters given by equations (9) and (10).

$$
\begin{aligned}
& \frac{1}{2} \phi\left(\frac{x}{2}\right)=\sum_{k-\infty}^{\infty} h(k) \phi(x-k) \\
& \frac{1}{2} \psi\left(\frac{x}{2}\right)=\sum_{k-\infty}^{\infty} g(k) \psi(x-k)
\end{aligned}
$$

1) Haar wavelet: The Haar wavelet was the first and the simplest example of an orthonormal wavelet transformation [26]. The Haar function $\psi(t)$ is a rectangular pulse described as

$$
\psi(t)=\left\{\begin{array}{cl}
1 & 0 \leq t<\frac{1}{2} \\
-1 & \frac{1}{2} \leq t<1 \\
0 & \text { otherwise }
\end{array}\right.
$$

The scale function $\phi(t)$ is given by

$$
\phi(t)= \begin{cases}1 & 0 \leq t<1 \\ 0 & \text { otherwise }\end{cases}
$$

2) EP distribution: The Exponential-Polynomial (EP) distribution [27] was made for Haar wavelet coefficients. It describes the difference between two independent random variables fitted by the Gamma distribution. For single-look SAR images $(L=1)$ the EP pdf is a Gamma convolved with the same Gamma, but with the inverted argument [31]. Also, since Exponential is a special case for a Gamma with $L=1$, the difference between two Gammas can be defined as a generalization of the Laplace distribution, i.e., the difference between two Exponentials [32]. Figure 1 illustrates the wavelet coefficients with the EP distribution considering one decomposition level with the Haar wavelet. The expression for the EP distribution is given by

$$
\begin{gathered}
p_{e p}(y)=\frac{\exp \left(-\frac{L}{b}|y|\right)}{(L-1) !}\left(\frac{L}{a}\right)^{L}\left(\frac{L}{b}\right)^{L} \\
{\left[\sum_{j=0}^{L-1} \frac{(L+j-1) !}{j !(L-j-1) !\left(\frac{L}{a}+\frac{L}{b}\right)^{L+j}}|y|^{L-j-1}\right]}
\end{gathered}
$$

where $a$ and $b$ are mean values of two different patches under the NLM concept. $p_{e p}(y)$ is called Exponential-Polynomial because it is a product of an exponential by a polynomial function. If a random variable follows the EP distribution, it is defined as $x \sim \operatorname{EP}(L, L / a, L / b)$.

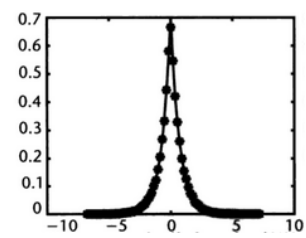

(a) Single-look

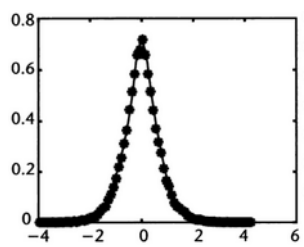

(b) Three looks

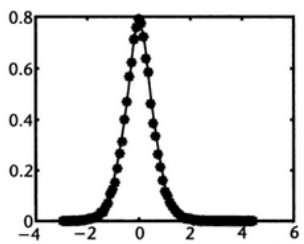

(c) Four looks

Fig. 1. Pdf of EP for different looks considering one decomposition level with the Haar wavelet.

By applying an $M$ decomposition level considering the Haar wavelet, the scale $c^{M}$ and detail $w^{M}$ coefficients were fitted by equations (14) and (15) [27].

$$
c^{M} \sim \Gamma\left(4^{M} L, 2^{-M}\right)
$$




$$
w^{M} \sim \operatorname{EP}\left(2^{2 M-1} L, 2^{-M} L\right)
$$

\section{NL-MEAnS AND THE Stochastic Distances}

The NLM [6] algorithm considers the patches similarity of an image. Patches are windows of $m \times m$ size centered on the pixels with $m \in \mathbb{N}^{*}$ and odd [4]. The NLM will use the Euclidean distance to compute a weighted average between these patches to measure the similarity. The goal of the proposed method is to replace the original Euclidean distance of the NLM filter by the new distances to turn the NLM able to filter the speckle noise in the wavelet domain. The family of divergences $(h, \phi)$ [33] was used to derive the stochastic distances integrals [14]: Kullback-Leibler, Rényi, Hellinger, Bhattacharyya, Jensen-Shannon, ArithmeticGeometric, Triangular and Harmonic Mean. Based on the integrals of these distances, we computed new distances for the EP distribution. In the following (16) to (23) formulas, $a_{1}$ and $a_{2}$ are means of two different patches.

Kullback-Leibler distance $\left(d_{K L E P}\right)$ :

$$
d_{K L E P}=\frac{\left(\ln \left(a_{1}+1\right)-\ln \left(a_{2}+1\right)\right)\left(a_{1}-a_{2}\right)}{2\left(a_{1}+1\right)\left(a_{2}+1\right)}
$$

Hellinger distance $\left(d_{H E P}\right)$ :

$$
d_{H E P}=\frac{a_{1}+a_{2}-2 \sqrt{a_{1}+1} \sqrt{a_{2}+1}+2}{\left(2 a_{1}+2\right)\left(a_{2}+1\right)}
$$

Rényi distance with $\beta=0.5\left(d_{R E P}\right)$ :

$$
d_{R E P}=-2 \ln \left(1-d_{H}\right)
$$

Bhattacharyya distance $\left(d_{B E P}\right)$ :

$$
d_{B E P}=-\ln \left(1-d_{H}\right)
$$

Jensen-Shannon $\left(d_{J S E P}\right)$ :

$$
\begin{array}{r}
d_{J S E P}=\frac{(\ln (2 \mathrm{a} 2+2)-\ln (\mathrm{a} 1+\mathrm{a} 2+2))}{2(\mathrm{a} 1+1)}+ \\
\frac{(\ln (2 \mathrm{a} 1+2)-\ln (\mathrm{a} 1+\mathrm{a} 2+2))}{2(\mathrm{a} 2+1)}
\end{array}
$$

Arithmetic-Geometric distance $\left(d_{A G E P}\right)$ :

$$
d_{A G E P}=-\frac{(\ln (\mathrm{a} 2+1)+\ln (4 \mathrm{a} 1+4)-2 \ln (\mathrm{a} 1+\mathrm{a} 2+2))(\mathrm{a} 1+\mathrm{a} 2+2)}{4(\mathrm{a} 1+1)(\mathrm{a} 2+1)}
$$

Triangular distance $\left(d_{T E P}\right)$ :

$$
d_{T E P}=\frac{\left(a_{1}-a_{2}\right)^{2}}{\left(a_{1}+1\right)\left(a_{2}+1\right)\left(a_{1}+a_{2}+2\right)}
$$

Harmonic-Mean distance $\left(d_{H M E P}\right)$ :

$$
d_{H M E P}=-\ln \left(1-\frac{d_{T}}{2}\right)
$$

According to equation (14) in Section II-A2, the approximation coefficient follows a Gamma distribution. Therefore, the distances for this distribution were also computed. However, for the eight stochastic distances, only four distances had their closed formulas found by equations (24) to (27).

Kullback-Leibler distance $\left(d_{K L G}\right)$ :

$$
d_{K L G}=\frac{\left(a_{1}-a_{2}\right)^{2}}{2 a_{1} a_{2}}
$$

Hellinger distance $\left(d_{H G}\right)$ :

$$
d_{H G}=\frac{\left(\sqrt{a_{1}}-\sqrt{a_{2}}\right)^{2}}{a_{1}+a_{2}}
$$

Rényi distance with $\beta=0.5\left(d_{R G}\right)$ :

$$
d_{R G}=-2 \ln \left(\frac{2 \sqrt{a_{1}} \sqrt{a_{2}}}{a_{1}+a_{2}}\right)
$$

Bhattacharyya distance $\left(d_{B G}\right)$ :

$$
d_{B G}=-\ln \left(\frac{2 \sqrt{a_{1}} \sqrt{a_{2}}}{a_{1}+a_{2}}\right)
$$

1) Distances proprieties: A distance $d(x, y)$ must be homogeneous, symmetrical and positive. Figures 2 and 3 show the good behavior of the stochastic distances obtained for the EP and Gamma distributions considering single-look speckle. Their plots are above the $x$-axis and the dark blue color indicates the zero value when $x=y$. Finally, the curves indicate symmetry when interchanging $x$ and $y$ values.

\section{DISCUSSION AND RESULTS}

The proposed algorithm is presented in Figure 4. The noisy image is decomposed with one level using the Haar wavelet, which gives the approximation coefficient (Ac) and the details coefficients: horizontal (Hc), vertical (Vc) and diagonal (Dc). Note that $\mathrm{L}$ indicates the low-pass filter while $\mathrm{H}$ denotes the high-pass filter. Then we apply the NLM filtering with the stochastic distances for Gamma and EP distributions and an inverse DWT (IDWT) is performed to obtain the filtered image. For the real and synthetic experiments below, the following stochastic distances were used: Kullback-Leibler for NLM with Gamma distribution and Jensen-Shannon for NLM with EP distribution.

The proposed filter was compared with FANS [13], OBNLM [34], Iterative PPB [10], SAR-BM3D [12]. Furthermore, for the mentioned algorithms, we chose the parameter settings proposed in the original papers.

\section{A. Real experiments}

We used two $512 \times 512$ real intensity SAR single-look scenes to compare and analyze the filters from the Oberpfaffenhofen region near Munich (Germany) taken through the ESAR platform presented in Figure 5. The NLM was set with a $5 \times 5$ patch and a $11 \times 11$ search window for all regions. The approximation coefficient of DWT was filtered with the smoothing parameter $h=5 \times 10^{-3}$. The detail coefficients were processed with $h=5 \times 10^{-3} \sigma_{c A}^{2}$, where $\sigma_{c A}^{2}$ is the approximation coefficient variance. All values were found experimentally.

Examining Figures 6 and 7, which show the filtered regions 1 and 2, we can consider that the proposed method is competitive with other filters. Visually, the PPB produces blurry images. Furthermore, our approach is quite effective in subtracting the 


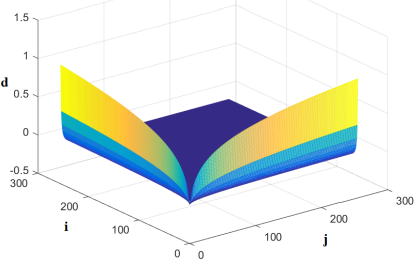

(a) Arithmetic-Geometric

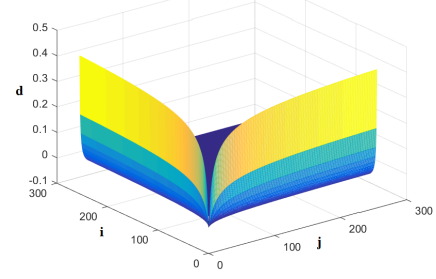

(c) Hellinger

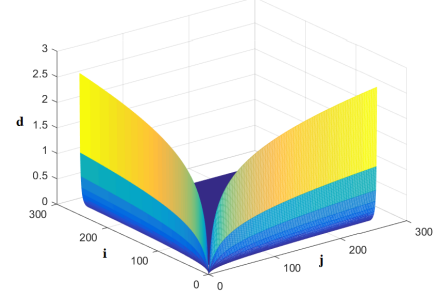

(e) Kullback-Leibler

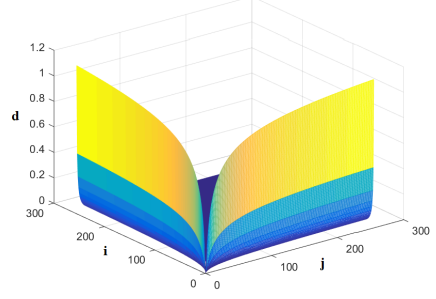

(g) Rény

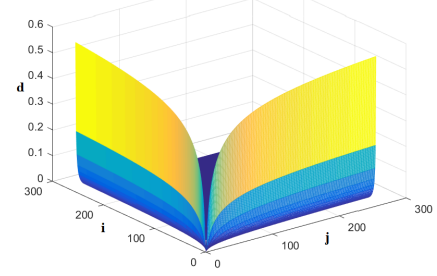

(b) Bhattacharyya

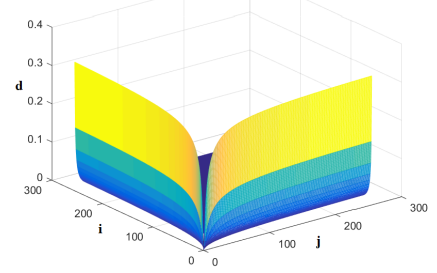

(d) Jensen-Shannon

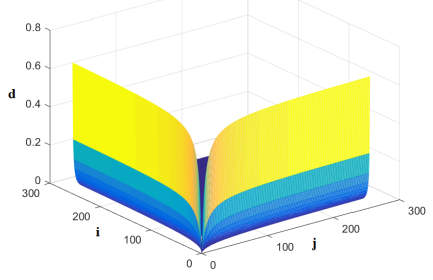

(f) Harmonic-Mean

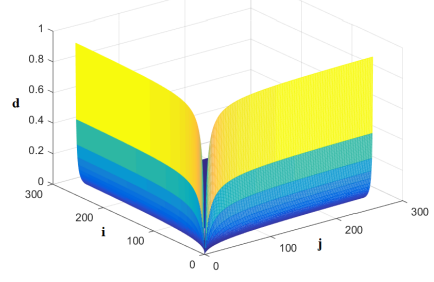

(h) Triangular

Fig. 2. Stochastic distances 3D plots for the EP distribution for single-look speckle.

speckle in homogeneous and heterogeneous regions. However, details are lost in extremely heterogeneous surfaces as described in the previous section. The FANS filter also removes fine details in heterogeneous areas.

Applying the ratio between the noisy image $Z$ and the filtered image $\hat{f}: r=Z / \hat{f}$ is an effective measure without reference [5]. This ratio shows the standard noise removed that it is supposed to be distributed according to the Gamma distribution. With an ideal filter, $r$ should contain only pure noise pattern. Otherwise, it will give an image with structures and edges [4]. By observing Figures 8 and 9, we confirm the loss of some structures with our filter, like PPB, OBNLM and FANS. Al-

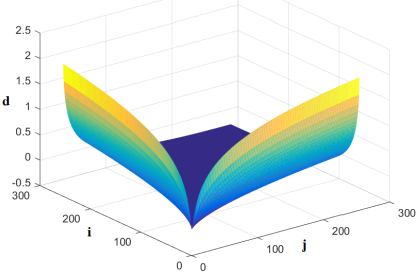

(a) Bhattacharyya

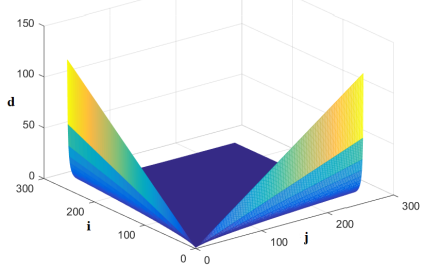

(c) Kullback-Leibler

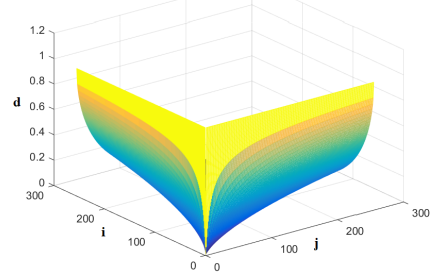

(b) Hellinger

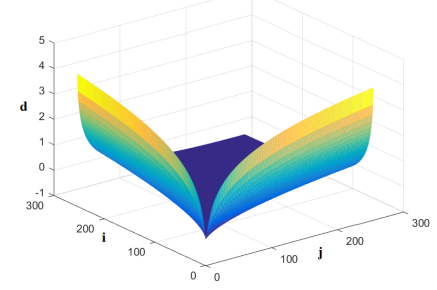

(d) Rény
Fig. 3. Stochastic distances 3D plots for the Gamma distribution for singlelook speckle.

though FANS demonstrates good results, it also loses details. The difficulty to find details on the SAR-BM3D ratio confirms its place as state-of-the-art. By using the mean $\left(\mu_{r}\right)$ and standard deviation $\left(\sigma_{r}\right)$ of the ratio $r$ it is possible to make a quantitative approach. The $\mu_{r}$ and $\sigma_{r}$ values should be close to the theoretical ones, i.e., $\mu \approx 1$ and $\sigma \approx 1$ for $L=1$. Tables I and II show the $\mu_{r}$ and $\sigma_{r}$ values for Regions 1, and 2. We note that the mean and the variance of the proposed method are the ones that most approached the ideal value. Although the PPB presents a blurred image, its $\sigma_{r}$ is superior to the other algorithms.

TABLE I

MEAN $\left(\mu_{r}\right)$ AND VARIANCE $\left(\sigma_{r}\right)$ OF REgION 1 RATIO. THE BEST RESUlts ARE MARKED IN BOLD.

\begin{tabular}{cccc}
\hline Filters & $\mu_{r}$ & $\sigma_{r}$ & Time (s) \\
\hline FANS & 0.83 & 0.46 & 6.43 \\
PM & $\mathbf{1 . 0 0}$ & $\mathbf{0 . 6 1}$ & 201.03 \\
OBNLM & 0.94 & 0.50 & 0.97 \\
PPB & 0.96 & 0.57 & 62.46 \\
SAR-BM3D & 0.96 & 0.42 & 99.73
\end{tabular}

TABLE II

MeAN $\left(\mu_{r}\right)$ AND VARIANCE $\left(\sigma_{r}\right)$ OF REgion 2 Ratio. THE BEST RESUlts ARE MARKED IN BOLD.

\begin{tabular}{cccc}
\hline Filters & $\mu_{r}$ & $\sigma_{r}$ & Time $(\mathrm{s})$ \\
\hline FANS & 0.83 & 0.46 & 6.83 \\
PM & $\mathbf{1 . 0 0}$ & $\mathbf{1 . 0 0}$ & 199.57 \\
OBNLM & 0.94 & 0.50 & 1.10 \\
PPB & 0.96 & 0.57 & 63.18 \\
SAR-BM3D & 0.96 & 0.44 & 103.11
\end{tabular}




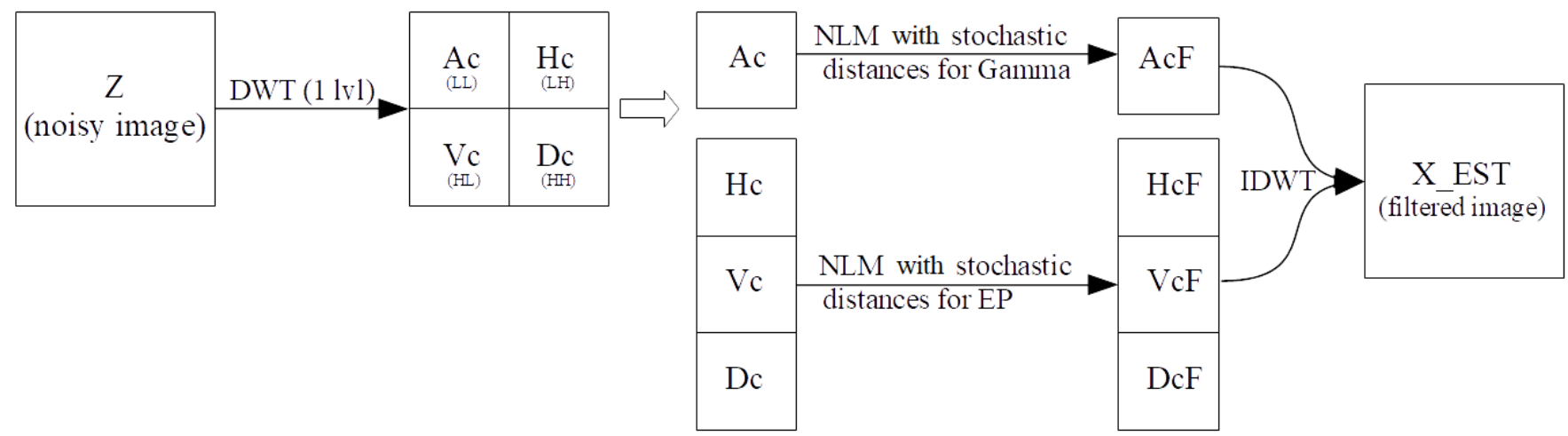

Fig. 4. Diagram of the proposed method with one level decomposition using the Haar wavelet.

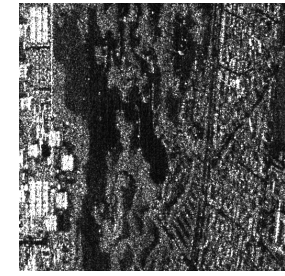

(a) Region 1

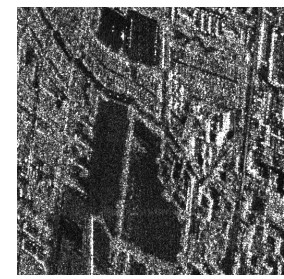

(b) Region 2
Fig. 5. Intensity HH SAR regions with $L=1$.

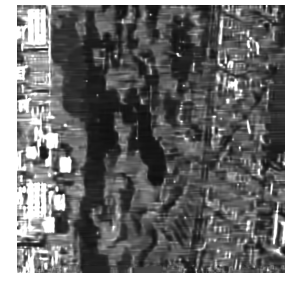

(a) FANS

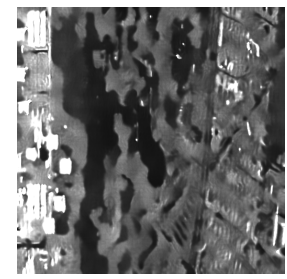

(c) PPB

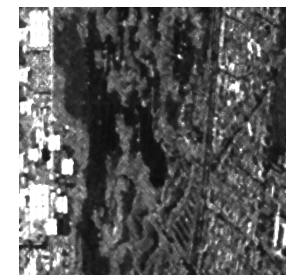

(b) OBNLM

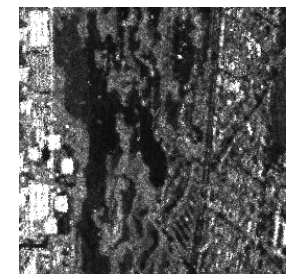

(d) PM

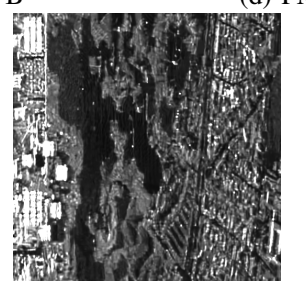

(e) SAR-BM3D
Fig. 6. Results for Region 1 (R1). PM: proposed method.

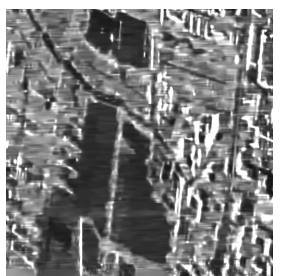

(a) FANS

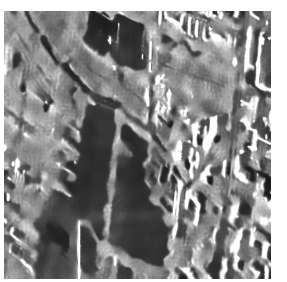

(c) PPB

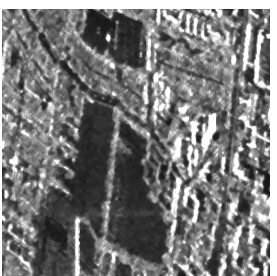

(b) OBNLM

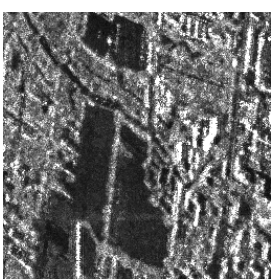

(d) PM

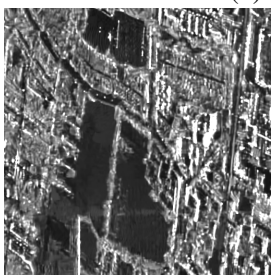

(e) SAR-BM3D

Fig. 7. Results for Region 2 (R2). PM: proposed method.

\section{CONClusion}

SAR imaging systems are important to study the terrestrial surface. They use coherent processing that generates the speckle noise. This noise needs be eliminated so that the SAR image can provide a good interpretation.

A technique to reduce single-look speckle noise in intensity SAR image was presented. Its approach is based on the NLM filter philosophy, which was originally developed for additive noise.

The fundamental step of our approach is to apply the NLM algorithm with the stochastic distances calculated for Gamma and EP distributions, replacing the Euclidean distance. Furthermore, the process has been developed in the Haar wavelet 


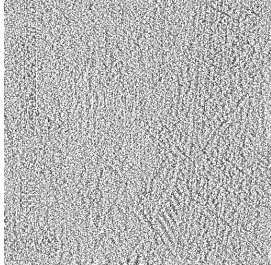

(a) FANS

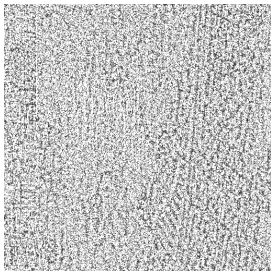

(c) PPB

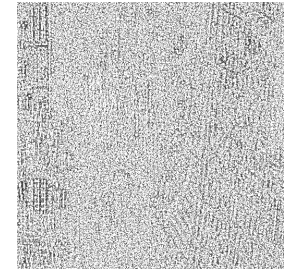

(b) OBNLM

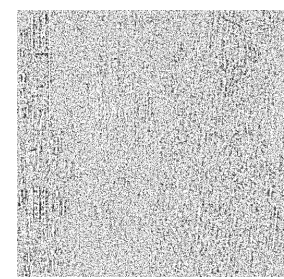

(d) PM

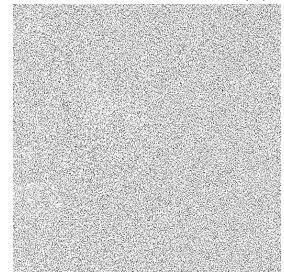

(e) SAR-BM3D

Fig. 8. Ratio for Region 1 (R1). PM: proposed method.

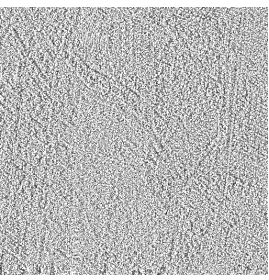

(a) FANS

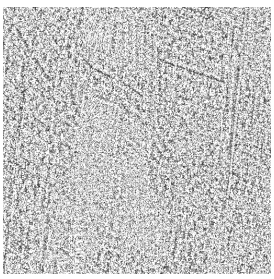

(c) PPB

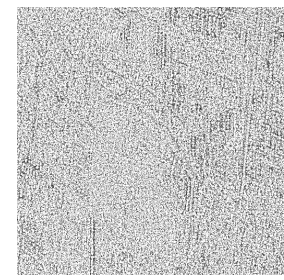

(b) OBNLM

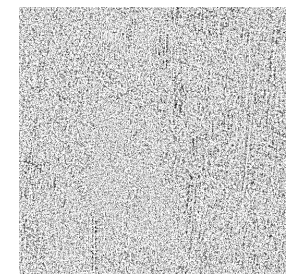

(d) PM

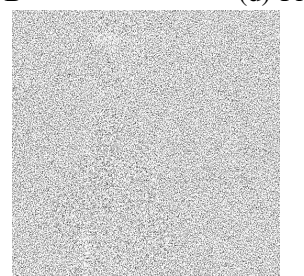

(e) SAR-BM3D

Fig. 9. Ratio for Region 2 (R2). PM: proposed method. domain

The real experiments of the proposed method were competitive with the other filters. It has been proven that the new approach is quite effective at removing the speckle in homogeneous regions. Also, it has been shown that there is a need to circumvent the problem of applying the proposed method in heterogeneous regions. However, the algorithm performance was higher than other filters primarily in removing the speckle in real SAR images.

Lastly, we can cite as a future work to apply the presented stochastic distances with the BM3D algorithm. We can also decrease the execution time of the proposed method by using an optimization of performance or vectorization code for NLM and propose the idea to other wavelets. Also, it is necessary to solve the loss of details in extremely heterogeneous regions.

\section{ACKNOWLEDGMENT}

The authors would like to thank the researchers who provided the filters code and also Professor Alejandro C. Frery who provided the ESAR image. This study was financed in part by the Coordenação de Aperfeiçoamento de Pessoal de Nível Superior - Brazil (CAPES) - Finance Code 001. The first author was supported by the CAPES scholarship and the work of the second author was supported by $\mathrm{CNPq}$, Brazil, through a scholarship under Grant Process 306742/2017-9.

\section{REFERENCES}

[1] P. Chatterjee and P. Milanfar, "Is denoising dead?" IEEE Transaction on Image Processing, vol. 19, no. 4, pp. 895-911, April 2010.

[2] A. Nath, "Image denoising algorithms: A comparative study of different filtration approaches used in image restoration," in 2013 International Conference on Communication Systems and Network Technologies (CSNT), 2013, pp. 157-163.

[3] C. A. Deledalle, L. Denis, S. Tabti, and F. Tupin, "Mulog, or how to apply gaussian denoisers to multi-channel sar speckle reduction?" IEEE Transactions on Image Processing, vol. 26, no. 9, pp. 4389-4403, September 2017.

[4] P. A. Penna and N. D. Mascarenhas, "(Non-) homomorphic approaches to denoise intensity sar images with non-local means and stochastic distances," Computers \& Geosciences, vol. 111, no. Supplement C, pp. 127 - 138, 2018. [Online]. Available: http://www.sciencedirect.com/science/article/pii/S0098300417300432

[5] F. Argenti, A. Lapini, T. Bianchi, and L. Alparone, "A tutorial on speckle reduction in synthetic aperture radar images," IEEE Geoscience and Remote Sensing Magazine, vol. 1, no. 3, pp. 6-35, 2013.

[6] A. Buades, B. Coll, and J. M. Morel, "A review of image denoising algorithm, with a new one," Multiscale Modeling and Simulation, vol. 4 , no. 2, pp. 490-530, 2005.

[7] K. Dabov, A. Foi, V. Katkovnik, and K. Egiazarian, "Image denoising by sparse 3-d transform-domain collaborative filtering," IEEE Transactions on Image Processing, vol. 16, no. 8, pp. 2080-2095, August 2007.

[8] M. Aharon, M. Elad, and A. Bruckstein, "K-svd: An algorithm for designing overcomplete dictionaries for sparse representation," IEEE Transactions on Signal Processing, vol. 54, no. 11, pp. 4311-4322, 2006.

[9] A. Efros and T. Leung, "Texture synthesis by non parametric sampling," Proceedings of the IEEE International Conference on Computer Vision, vol. 2, pp. 1033-1038, 1999.

[10] C. Deledalle, L. Denis, and F. Tupin, "Iterative weighted maximum likelihood denoising with probabilistic patch-based weights," IEEE Transactions on Image Processing, vol. 18, no. 12, pp. 2661-2672, December 2009.

[11] C. Deledalle, L. Denis, F. Tupin, A. Reigber, and M. Jäger, "Nlsar: A unified nonlocal framework for resolution-preserving (pol)(in)sar denoising," IEEE Transactions on Geoscience and Remote Sensing, vol. 53, no. 4, pp. 2021-2038, April 2015. 
[12] S. Parrilli, M. Poderico, C. Angelino, and L. Verdoliva, "A nonlocal sar image denoising algorithm based on llmmse wavelet shrinkage," IEEE Transactions on Geoscience and Remote Sensing, vol. 50, no. 2, pp. 606-616, February 2012.

[13] D. Cozzolino, S. Parrilli, G. Scarpa, G. Poggi, and L. Verdoliva, "Fast adaptive nonlocal sar despeckling," IEEE Geoscience and Remote Sensing Letters, vol. 11, no. 99, pp. 1-5, 2013.

[14] A. D. C. Nascimento, R. J. Cintra, and A. C. Frery, "Hypothesis testing in speckled data with stochastic distances," IEEE Transactions on Geoscience and Remote Sensing, vol. 48, no. 1, pp. 373-385, January 2010.

[15] P. A. A. Penna and N. D. A. Mascarenhas, "Sar speckle nonlocal filtering with statistical modeling of Haar wavelet coefficients and stochastic distances," IEEE Transactions on Geoscience and Remote Sensing, vol. 57, no. 9, pp. 7194-7208, September 2019.

[16] L. Torres, T. Cavalcante, and A. C. Frery, "Speckle reduction using stochastic distances," in Progress in Pattern Recognition, Image Analysis, Computer Vision, and Applications, ser. Lecture Notes in Computer Science, L. Alvarez, M. Mejail, L. Gomez, and J. Jacobo, Eds., vol. 7441. Springer Berlin Heidelberg, 2012, pp. 632-639.

[17] L. Torres and A. C. Frery, "Sar image despeckling algorithms using stochastic distances and nonlocal means," in Workshop of Theses and Dissertations (WTD) in SIBGRAPI 2013 (XXVI Conference on Graphics, Patterns and Images), Arequipa, Peru, August 2013.

[18] L. Torres, S. J. Sant'Anna, C. da Costa Freitas, and A. C. Frery, "Speckle reduction in polarimetric $\{\mathrm{SAR}\}$ imagery with stochastic distances and nonlocal means," Pattern Recognition, vol. 47, no. 1, pp. $141-157,2014$. [Online]. Available: http://www.sciencedirect.com/science/article/pii/S0031320313001532

[19] C. A. N. Santos, D. L. N. Martins, and N. D. A. Mascarenhas, "Ultrasound image despeckling using stochastic distance-based BM3D," IEEE Transactions on Image Processing, vol. 26, no. 6, pp. 2632-2643, June 2017.

[20] C. A. N. Santos and N. D. A. Mascarenhas, "Geodesic distances in probabilistic spaces for patch-based ultrasound image processing," IEEE Transactions on Image Processing, vol. 28, no. 1, pp. 216-226, January 2019.

[21] C. A. Santos and N. D. Mascarenhas, "Patch similarity in ultrasound images with hypothesis testing and stochastic distances," Computerized Medical Imaging and Graphics, 2019. [Online]. Available: http: //www.sciencedirect.com/science/article/pii/S089561111930028X

[22] A. A. Bindilatti and N. D. A. Mascarenhas, "A nonlocal poisson denoising algorithm based on stochastic distances," IEEE Signal Processing Letters, vol. 20, no. 11, pp. 1010-1013, November 2013.

[23] A. A. Bindilatti, M. A. Vieira, and N. D. Mascarenhas, "Poisson Wiener filtering with non-local weighted parameter estimation using stochastic distances," Signal Processing, vol. 144, no. Supplement C, pp. 68 - 76, 2018. [Online]. Available: http://www.sciencedirect.com/ science/article/pii/S0165168417303523

[24] S. Mallat, "A theory for multiresolution signal decomposition: the wavelet representation," Pattern Analysis and Machine Intelligence, IEEE Transactions on, vol. 11, no. 7, pp. 674-693, July 1989.

[25] A. Misra, B. Kartikeyan, and S. Garg, "Wavelet based sar data denoising and analysis," in Advance Computing Conference (IACC), 2014 IEEE International, February 2014, pp. 1087-1092.

[26] R. S. Stankovic and B. J. Falkowski, "The haar wavelet transform: its status and achievements," Computers \& Electrical Engineering, vol. 29, no. 1, pp. 25 - 44, 2003. [Online]. Available: http: //www.sciencedirect.com/science/article/pii/S0045790601000118

[27] A. K. Chan and C. Peng, Wavelet for sensing technologies. Boston : Artech House, 2003.

[28] J. W. Goodman, "Some fundamental properties of speckle*," J. Opt. Soc. Am., vol. 66, no. 11, pp. 1145-1150, November 1976. [Online]. Available: http://www.osapublishing.org/abstract.cfm?URI= josa-66-11-1145

[29] H. H. Arsenault and G. April, "Properties of speckle integrated with a finite aperture and logarithmically transformed," J. Opt. Soc. Am., vol. 66, no. 11, pp. 1160-1163, November 1976. [Online]. Available: http://www.osapublishing.org/abstract.cfm?URI=josa-66-11-1160

[30] E. S. Ribeiro, "Novas propostas em filtragem de projeções tomográficas sob ruído poisson," Master's thesis, Federal University of São Carlos Computing Department, 2010.

[31] B. Klar, "A note on gamma difference distributions," Journal of Statistical Computation and Simulation, vol. 85 , no. 18, pp. $3708-$
3715, 2015. [Online]. Available: http://dx.doi.org/10.1080/00949655. 2014.996566

[32] E. Krishna and K. Jose, "Marshall-olkin generalized asymmetric laplace distributions and processes," Statistica, vol. 71, no. 4, pp. 453-467, 2011. [Online]. Available: https://rivista-statistica.unibo.it/article/view/3627

[33] M. Salicru, D. Morales, M. Menendez, and L. Pardo, "On the applications of divergence type measures in testing statistical hypotheses," Journal of Multivariate Analysis, vol. 51, no. 2, pp. 372 391, 1994. [Online]. Available: http://www.sciencedirect.com/science/ article/pii/S0047259X84710682

[34] P. Coupé, P. Hellier, C. Kervrann, and C. Barillot, "Nonlocal meansbased speckle filtering for ultrasound images," IEEE Transactions on Image Processing, vol. 18, no. 10, pp. 2221-2229, 2009. 